\title{
Distinct Product Count
}

National Cancer Institute

\section{Source}

National Cancer Institute. Distinct Product Count. NCI Thesaurus. Code C104442.

The determination of the number of distinct products. 